\title{
Regards théoriques sur la perspective actionnelle dans l'enseignement des langues en France
}

The learning of foreign languages in France: the underlying theories of the action-oriented approach

\section{Margaret Bento}

\section{OpenEdition}

\section{Journals}

Édition électronique

URL : http://journals.openedition.org/educationdidactique/1404

DOI : 10.4000/educationdidactique.1404

ISSN : 2111-4838

\section{Éditeur}

Presses universitaires de Rennes

\section{Édition imprimée}

Date de publication : 7 février 2013

Pagination : 87-100

ISBN : 978-2-7535-2261-9

ISSN : 1956-3485

\section{Référence électronique}

Margaret Bento, « Regards théoriques sur la perspective actionnelle dans l'enseignement des langues en France », Éducation et didactique [En ligne], 7-1 | 2013, mis en ligne le 31 janvier 2015, consulté le 10 décembre 2020. URL : http://journals.openedition.org/educationdidactique/1404 ; DOI : https://

doi.org/10.4000/educationdidactique.1404 


\section{REGARDS THÉORIQUES SUR LA PERSPECTIVE ACTIONNELLE DANS L'ENSEIGNEMENT DES LANGUES EN FRANCE}

Margaret Bento

EDA, Université Paris Descartes, Paris

Le Cadre européen commun de référence pour les langues, document de référence sur la conception de l'enseignement des langues en Europe, se situe dans une perspective actionnelle. Cependant, il ne promeut aucune théorie sousjacente. Cet article, à partir de la métasynthèse qualitative de la littérature sur la question, montre que les chercheurs en didactique et les didacticiens des langues orientent la perspective actionnelle vers les théories constructiviste et socioconstructiviste.

Mots-clés : CECRL, perspective actionnelle, constructivisme, socio-constructivisme, métasynthèse

The learning of foreign languages in France : the underlying theories of the action-oriented approach

The Common European Framework of Reference for Languages is a document which provides a practical tool to learn languages in Europe and adopts an action-oriented approach. However, it is not attached to any competing linguistic or educational theories. This article shows that despite of this, researchers in language teaching impact the action-oriented approach on constructivist and social constructivist theories.

Keywords: CEFR, action-oriented approach, constructivism, social constructivism, metasynthesis 
Margaret Bento

\section{INTRODUCTION}

Le Cadre européen commun de référence pour les langues (dorénavant CECRL ou Cadre) (Conseil de l'Europe, 2001) constitue le document de référence sur la conception de l'enseignement des langues en Europe aujourd'hui. Les auteurs du Cadre indiquent se situer dans une "perspective actionnelle en considérant avant tout l'usager et l'apprenant d'une langue comme des acteurs sociaux ayant à accomplir des tâches (qui ne sont pas seulement langagières) dans des circonstances et un environnement donné, à l'intérieur d'un domaine d'action particulier. Si les actes de parole se réalisent dans des activités langagières, celles-ci s'inscrivent elles-mêmes à l'intérieur d'actions en contexte social qui seules leur donnent leur pleine signification. » (Conseil de l'Europe, 2001, p. 15). Cependant, le CECRL ne revendique aucune théorie sous-jacente au niveau méthodologique puisque « à l'heure actuelle, il n'y a pas de consensus fondé sur une recherche assez solide en ce qui concerne cette question pour que le cadre européen lui-même se fonde sur une quelconque théorie de l'apprentissage. " (Conseil de l'Europe, 2001, p. 108). Le CECRL se veut donc « non dogmatique : il n'est rattaché de manière irrévocable et exclusive à aucune des théories ou pratiques concurrentes de la linguistique ou des sciences de l'éducation. » (Conseil de l'Europe, 2001, p. 14). S'il n'y a pas d'opération d'élaboration méthodologique de la perspective actionnelle par le CECRL avec des fondements scientifiques posés a priori, en revanche, on peut se poser la question de savoir s'il existe une opération d'étayage scientifique réalisée a posteriori par les didacticiens. Quelle est la position des didacticiens qui écrivent sur la question et qui diffusent ainsi leur lecture de la perspective actionnelle? Dans cet article, je propose une métasynthèse de la littérature sur la question afin de repérer les courants théoriques mobilisés par les didacticiens.

\section{MÉTHODOLOGIE}

Ce travail a pour cadre méthodologique la métasynthèse. Finfgeld (2003) détermine trois types de métasynthèse. Le premier consiste en un travail de synthèse afin d'élaborer une nouvelle théorie sur un phénomène et de dépasser les limites théoriques actuelles. Dans le deuxième type de métasynthèse, il s'agit de déconstruire puis de reconstruire et enfin de synthétiser des concepts abstraits afin de reconceptualiser l'interprétation du phénomène étudié. Enfin, le troisième type consiste en une description. Il s'agit d'une synthèse de résultats de recherches qualitatives avec pour objectif d'offrir une analyse compréhensive d'un phénomène donné. C'est dans cette dernière perspective que ce travail se situe. Il s'agira donc de mettre en évidence les éléments adéquats pour répondre à notre questionnement : quelles sont les théories mobilisables pour étayer les approches actionnelles d'après les didacticiens des langues?

Sandelowski et Barroso (2006) proposent comme technique d'analyse la comparaison à visée constante (constant targeted comparison) que nous retiendrons ici. Cette technique permet de déterminer les similarités et les différences des éléments ciblés afin d'en clarifier les caractéristiques. Il ne s'agit pas de mettre des résultats ensemble et d'en présenter une grande quantité comme on pourrait le voir dans une simple revue de la littérature mais plutôt de dégager des éléments pertinents et adaptés à une question. Il n'existe pas à ma connaissance de type spécifique de métasynthèse en didactique des langues, aussi j’ai emprunté l'approche pratiquée par les anthropologues Noblit et Hare dans leur ouvrage Metaethnography: Synthesizing Qualitative Studies ${ }^{1}$ (1988, p. 26-29). Chaque étude a été lue afin d'identifier les éléments (mots-clés, phrases, références) liés à des théories d'apprentissage, sociologiques et linguistiques. Puis, à partir de la liste des mots/ phrases clés établis, des regroupements ont été effectués afin de dégager de nouvelles interprétations. La dernière étape consistait à faire une synthèse des informations retenues afin d'en proposer une lecture claire.

Pour la recherche qui nous concerne, la procédure a commencé par une revue de la littérature sur la perspective actionnelle en France entre 2001 et 2011, 2001 constituant un point de départ avec l'édition du CECRL. Des mots-clés ont été retenus lors d'un premier recueil de données : perspective actionnelle, approche actionnelle, CECRL. Ce choix a été motivé par le fait que perspective actionnelle et approche actionnelle constituent les deux expressions en usage sur la question et le CECRL représente le document de référence sur le sujet. Les écrits portant sur d'autres pays que la France ont été écartés. La littérature retenue était donc celle qui présentait des éléments théoriques et ou descriptifs sur la perspec- 
tive actionnelle dans l'enseignement/apprentissage des langues étrangères en France.

Les critères d'exclusion étaient ceux qui n'intégraient pas les caractéristiques citées ci-dessus et les études dont la source ou l'année n'étaient pas identifiées (cela concernait exclusivement des documents internet).

Sur 65 textes recueillis au départ, 41 ont été sélectionnés. Le corpus se composait d'articles, d'ouvrages et de thèses issus de la didactique des langues (français, anglais, espagnol). Le détail du corpus se trouve dans l'annexe.

\section{LES RÉSULTATS}

L'analyse des études sélectionnées a révélé plusieurs éléments en lien avec la théorie. Trois grands champs théoriques ont pu être mis au jour : les théories de l'apprentissage, les sciences du langage et la sociologie.

\section{Les théories de l'apprentissage}

Dans le corpus, les approches actionnelles sont associées au constructivisme, au socioconstructivisme et aux théories de l'activité. Les citations, les annotations et leurs recoupements ont permis de mettre en évidence quatre concepts : la tâche, la coopération ou co-action/communic-action, le conflit sociocognitif et la métacognition.

\section{La notion de tâche}

La perspective actionnelle est associée à la notion de tâche issue du concept anglo-saxon de Task-Based Learning (Ellis 2003 ; Nunan 2004). Pour expliquer cette notion, les auteurs reprennent le plus souvent les termes du CECRL qui la définit comme «toute visée actionnelle que l'acteur se représente comme devant parvenir à un résultat donné en fonction d'un problème à résoudre, d'une obligation à remplir, d'un but qu'on s'est fixé. » (Conseil de l'Europe, 2001, p. 16). Cependant, Puren (2003, 2004) donne une définition de la tâche volontairement abstraite afin qu'elle puisse être utilisée dans tous les environnements mais il précise que « [1]'orientation' d'une tâche est déterminée en fonction de son critère premier de réussite. »(Puren, 2004, p. 15). Ainsi pour l'auteur il faut parler de tâche 'orientée action'. Bérard (2009, p. 38), quant à elle, souligne l'existence des deux acceptions du terme " tâche », celle qui englobe toutes les activités d'apprentissage de langue et celle de tâche-problème.

Selon la plupart des auteurs, pour qu'une tâche soit actionnelle, elle doit répondre à un certain nombre de critères.

- Les tâches proposées aux élèves ne doivent pas uniquement se focaliser sur les actions verbales (tâches langagières), à l'instar du Task-Based Learning, mais doivent également prendre en compte les actions non-verbales (tâches extralangagières) ${ }^{2}$. Médioni (2008) définit la tâche comme une activité contextualisée présentant une situation de la vie dont l'objectif est extralinguistique. Richer (2009) précise que les interactions lient le langage et l'action (Roulet, 1999 ; Filliettaz, 2002) : « l'action génère le langage et le langage appelle en retour des réponses physiques. » (Richer, 2009, p. 27). Bourguignon (2006, p. 64) précise qu'il s'agit de « préparer les apprenants à utiliser la langue dans des situations imprévues. Ainsi, les stratégies cognitives développées ne devront pas être uniquement au service de l'apprentissage de la langue sur un axe vertical (consistant à aborder des textes structurés de manière toujours plus complexe) mais aussi au service de l'action sur un axe horizontal, c'est-àdire pour lui permettre d'aborder des situations de plus en plus complexes (contexte plus ou moins familier, interlocuteurs plus ou moins stéréotypés, objectif de l'action plus ou moins ciblé...) » Pour Springer (2009, p. 32), il s'agit d'intégrer à la fois des compétences langagières et des compétences générales et sociales dans les dispositifs d'évaluation. Ainsi, une prise en compte générale du contexte s'avère nécessaire.

- Les tâches doivent présenter une résolution de problème au sens large. Auger et Vincent (2009, p. 103) souligne qu'un problème implique à la fois la question à résoudre, prêtant à discussion, et la difficulté à surmonter. Le problème constituerait la « représentation qu'a un sujet de l'écart entre la situation de départ, jugée insatisfaisante, et une situation désirée, perçue comme plus satisfaisante, mais pour laquelle il ne possède pas de procédure permettant de le réduire, alors que la tâche correspond à ce que devra faire ce sujet dans le cadre de 
cette situation problématique. » Il s'agit pour les élèves de mobiliser des savoirs et savoir-faire afin de résoudre les tâches-problèmes à accomplir ce qui permettra de restructurer ce qu'ils savent déjà mais également d'acquérir de nouveaux savoirs et savoir-faire.

- Plusieurs auteurs précisent que la tâche doit s'inscrire dans une logique de conceptualisation, de réflexion sur la langue (Chini, 2008, p. 15 ; Goutéraux, 2008, p. 19-21; Hamez et Lepez, 2010, p. 55 ; Médioni, 2008, p. 14-15).

- Les tâches actionnelles doivent viser l'authenticité. Une tâche est « [...] un acte social en soi : elle présente une situation de la vie réelle, connue, voire familière $[\ldots]$ : décider d'un rendez-vous, chanter une chanson, écrire au courrier des lecteurs, écouter un conte, réaliser une devinette,... Inversement, faire un exercice, remplir une fiche de compréhension, employer le subjonctif imparfait ne sont pas des actes sociaux. " (Médioni, 2008 , p. 8). La tâche est ainsi « orientée vers un but, impose stratégie et planification, a une pertinence et un sens, implique de ce fait l'apprenant, offre toute mise en œuvre des ressources disponibles, définit clairement un résultat communicatif identifiable, est réaliste et faisable. » (Springer, 2009, p. 30 ; cf. aussi Ellis, 2003) afin de dépasser la simple simulation. Cependant, pour certains il faut aller encore plus loin en passant par des tâches ancrées dans la vie réelle ce que semblent permettre les TICE (Mangenot et Penilla, 2009 ; Ollivier, 2009). Les documents authentiques sont ainsi privilégiés (littérature, journaux, revues, émissions de radio et de télévision, documents internet, reproduction photographique, documents produits par les élèves eux-mêmes pour réaliser leur projet...) (en particulier Puren, 2002, p. 59 ; Nissen, 2003, p. 23 ; Perrichon, 2008, p. 136).

Beaucoup d'auteurs voient le projet comme une mise en œuvre possible de la tâche ou des microtâches. Puren (2009, p. 132) fait la distinction entre projets simulés et projets réels : « Même si les projets simulés resteront nécessaires, la perspective de l'agir social amène à privilégier les projets réels [...] éventuellement en combinaison avec les premiers. L'intérêt des projets simulés pour les auteurs d'un manuel est bien sûr qu'ils peuvent les contrôler de bout en bout, depuis la conception et la préparation jusqu'à sa réalisation et son exploitation, les projets réels impliquant nécessairement une plus grande autonomie chez les apprenants. » [Richer (2009, p. 39-40), quant à lui, insiste sur la différence entre tâche et projet. La tâche vise à atteindre un objectif contrôlé par l'enseignant tandis que le projet se situe dans une logique de production (on apprend en faisant). Cependant, à l'instar de Piaget, l'ensemble des auteurs sont d'accord pour dire que les apprenants vont construire leurs connaissances sur la langue grâce à leur activité. L'élève est acteur $\mathrm{du}$ processus de connaissance et il organise son monde au fur et à mesure qu'il apprend. Il s'approprie les savoirs et les savoir-faire en les construisant à travers l'accomplissement des projets tout en mettant en œuvre les éléments déjà connus (par exemple connaissances énonciatives acquises en langue maternelle, dans leur(s) autre(s) langue(s), connaissances extra-linguistiques, etc.) (Goutéraux, 2008).

Bourguignon (2006, 2009 entre autres) introduit le terme de "scénario » comme un guidage pour mener à bien un projet. Il s'agit de prévoir l'enchaînement des tâches communicatives contrairement à Puren (2009) qui souligne que l'activité de référence n'est pas communicative.

\section{La co-action ou communic-action}

La tâche dans une perspective actionnelle est vue comme une action à la fois individuelle et collective. Tous mettent la communication interactive au centre des apprentissages. Certains introduisent à la suite de Puren (2002, 2004, 2006 entre autres) l'idée de co-action plutôt que d'interaction. Il s'agit de passer de parler avec les autres à agir avec les autres ou communiquer pour agir. Bourguignon (2006, 2009 entre autres) quant à elle préfère introduire le terme de communic-action. « Il s'agit donc bien de réaliser une tâche en langue étrangère, mais avant tout, de la réaliser ensemble. » (Nissen, 2003, p. 30).

Deux types d'interaction peuvent être relevés dans le corpus : l'interaction entre les apprenants et l'enseignant (expert) et l'interaction entre apprenants. Les connaissances des apprenants se développent sous l'influence de l'entourage. L'idée de coopération est dominante dans le corpus et constitue pour les auteurs le point fort de cette approche largement associée au modèle socioconstructiviste et à ses concepts : la zone proximale de développement, le processus d'étayage, la métacognition, le conflit sociocognitif. Les trois premiers concepts impliquent 
plutôt des interactions de type dissymétrique (apprenant/expert) alors que le conflit sociocognitif se manifeste lors des interactions dissymétriques et symétriques (entre pairs).

Plusieurs auteurs du corpus (entre autres Catroux 2006, p. 7-8 ; Champion 2009, p. 149 ; Médioni 2010, p. 11-12 ; Nissen 2003, p. 55-75 et 305) s'appuient sur les travaux socioconstructivistes et notamment ceux de Vygotski, qui indiquent qu'un élève apprend plus vite et mieux lorsqu'il est accompagné d'un expert que seul (cf. aussi Vygotski, 1985 et Moscovici, 1984). Vygotski (1985) a défini la notion de zone proximale de développement ou ZPD qui correspond à l'écart entre la capacité de résolution de problèmes en collaboration avec un expert et celle atteinte par l'enfant seul. La ZPD représente ce que l'élève arrive à réaliser avec l'adulte et non en activité autonome. Ce que l'élève effectue avec un autre dans un premier temps, il sera capable de le faire seul dans un deuxième temps. Ainsi le développement de l'élève irait du social à l'individuel. Les interactions sociales que ce soit entre pairs ou entre apprenant et enseignant permettent de construire des compétences cognitives.

L'étayage constitue un autre concept associé à l'apprentissage coopérant par certains auteurs du corpus (entre autres, Hamez et Lepez 2009, p. 55 ; Médioni 2010, p. 12 ; Nissen 2003, p. 57-75 et 145). Le processus d'étayage, mis en évidence par Bruner (1983), désigne la mise en place de formats (formes régulatrices des échanges) par un enseignant qui va guider l'élève. L'enseignant va montrer à l'élève les chemins possibles pour effectuer la tâche (sans la résoudre à sa place) et présenter des modèles de solution. «L'expert reconnaît une zone proximale de développement chez le novice et ajuste son comportement dans cette action conjointe. » (Nissen, 2003, p. 57). Il s'agit aussi de maintenir l'intérêt et la motivation de l'élève dans la tâche qu'il doit effectuer. En même temps, il faut éviter toute forme de dépendance. L'objectif au terme du processus d'étayage est l'autonomisation de l'élève dans la résolution des problèmes (Médioni 2010, p. 12). Pour Médioni (2009, p. 141) cet étayage peut s'opérer entre pairs et permet « la confrontation des représentations plus facilement que dans un " dialogue » enseignant-élève : l'élève peut ne pas oser faire part de ses conceptions, ses croyances, ses opinions, à un enseignant détenteur, à ses yeux, d'un savoir abouti. Il peut davantage prendre ce risque face à ses pairs. »
Enfin, la notion de conflit sociocognitif développée par Perret-Clermont (1996), Doise et Mugny (1981) est relativement peu présente dans le corpus. Il s'agit de faire émerger une situation-problème, entre les élèves ou entre les élèves et l'enseignant, qu'ils seront ensuite amenés à résoudre ensemble. Les divergences entre l'élève et l'enseignant et surtout entre élèves vont conduire à une décentration par rapport au propre point de vue de chacun et permettre ainsi d'envisager le problème différemment. « Dans la dynamique du conflit socio-cognitif, la coopération doit ainsi être active, ce qui signifie que chacun des partenaires doit accepter de prendre en compte la réponse ou le point de vue de l'autre et de rechercher, sans esquiver le conflit cognitif (et également sans le résoudre au niveau relationnel), la construction d'une réponse commune. La simple soumission ou l'imposition d'un des points de vue signifierait une résolution du conflit au niveau relationnel et non au niveau cognitif, qui n'ont pas la même incidence sur le développement mental. » (Nissen, 2003, p. 64-65). Cependant, à l'instar de Gilly (2001), Nissen (2003, p. 96) insiste sur le fait qu'il peut y avoir co-élaboration acquiescante sans passer par un conflit. Il s'agit pour un élève d'approuver ce que propose un autre élève sans qu'il y ait désaccord. Quoi qu'il en soit, encore une fois, l'idée d'interaction sociale est importante, qu'elle donne lieu à un conflit ou pas.

Pour Puren (2002, p. 7 ; 2007, p. 7-8), le concept de « co-action » n'est pas nouveau puisqu'il était déjà mis en œuvre dans la « pédagogie du projet ». D’après lui, cette réactivation s'explique par la progression de l'intégration européenne : «[d]ans la méthodologie traditionnelle on formait un « lecteur » en le faisant traduire (des documents), dans la méthodologie active on formait un « commentateur» en le faisant parler sur (des documents); dans l'approche communicative on formait un « communicateur » en créant des situations langagières pour le faire parler avec (des interlocuteurs) et agir sur (ces mêmes interlocuteurs); dans la perspective actionnelle esquissée par le Cadre européen commun de référence [...] on se propose de former un «acteur social»; ce qui impliquera nécessairement, si l'on veut continuer à appliquer le principe fondamental d'homologie entre les fins et les moyens, de le faire agir avec les autres pendant le temps de son apprentissage en lui proposant des occasions de «co-actions» dans le sens d'actions communes à finalité collective. C'est cette 
dimension d'enjeu social authentique qui différencie la co-action de la simulation, technique de base utilisée dans l'approche communicative pour créer artificiellement en classe des situations de simple interaction langagière entre apprenants. »

La métacognition renvoie à la capacité qu'à l'élève à réfléchir sur la résolution d'un problème ou la manière d'effectuer une tâche. Catroux (2006, p. 4) souligne, en citant Narcy-Combes et Walski (2004, p. 35) que « [1] a tâche incite naturellement les apprenants à entrer activement dans la langue, à comprendre leurs propres processus d'apprentissage et, pour y parvenir, à se poser constamment des questions sur leur fonctionnement à partir des exemples repérés. » On ne s'attache donc pas uniquement au résultat final mais aussi au processus de résolution de la tâche. Pour Griggs (2009, p. 84) « il faudrait non seulement considérer la communication comme la finalité de l'apprentissage en tant qu'instrument de l'action humaine mais la traiter aussi comme une action en elle-même en prenant en compte sa dimension cognitive [...] L'approche actionnelle doit s'appuyer [...] sur une théorie cognitive permettant de cerner les mécanismes psycholinguistiques de la compréhension, de la production et de l'apprentissage de ces processus. » C'est l'articulation entre la pratique signifiante de la langue et les activités métalinguistiques et métacognitives qui va permettre de structurer l'apprentissage. «[...] c'est la démarche qui permet à l'élève de s'instaurer progressivement comme analyseur puis évaluateur, non seulement du fonctionnement de la langue et de la culture qui la sous-tend, mais aussi de sa propre pratique dans cette langue, celle qui lui permet de prendre conscience qu'en classe, l'objectif ne réside pas seulement dans le résultat de l'activité mais dans le processus lui-même, ce qui loin de " chosifier » la langue lui donne toute sa valeur. " (Chini, 2008, p. 14-15).

Les concepts autour des théories de l'apprentissage mis en évidence par les auteurs du corpus présentent une grande cohérence entre eux et convergent tous vers le constructivisme et le socioconstructivisme. Pour les auteurs, la cognition et l'interaction sont au cour de l'approche actionnelle. Dans cette perspective, Bange (2005, p. 8) pose deux principes : "Selon le premier, l'apprentissage est une affaire dont le sujet actif est l'individu apprenant. Je me risque à lâcher un gros mot : c'est une affaire de cognition. Le second principe pose que l'interaction entre le novice et un tuteur permet seule l'apprentissage. Ainsi est défini un cadre socio-cognitif pour l'apprentissage. »

\section{Les sciences du langage}

Si l'ensemble des auteurs du corpus donne des éléments sur les théories de l'apprentissage, les commentaires autour des théories linguistiques sont plus rares. Les chercheurs se centrent plutôt sur les processus d'apprentissage et sur les apprenants. Seuls quatre textes font clairement référence aux sciences du langage : Chini (2008), Goutéraux (2008), Richer (2005, 2009), Soubrié (2009).

Soubrié (2009) et Richer $(2005,2009)$ inscrivent les genres de discours dans l'approche actionnelle. Pour Soubrié (2009, p. 74), « il n'est plus seulement question, pour un acte de parole donné, de savoir sélectionner la réalisation linguistique appropriée à la situation de communication, mais de connaître également, sur un plan plus global, les contraintes thématiques, stylistiques et structurelles liées aux différents domaines de l'activité humaine, c'est-à-dire ce que l'on appelle les genres. » Pour Richer (2005, 2009), les genres de discours tels qu'ils ont été définis par Bakhtine (1979) puis par Bronckart (1996) et Maingueneau (1998) « constituent des réglages qui [...] opèrent à cinq niveaux constitutifs de la totalité textuelle :

- au niveau socio-énonciatif-pragmatique : tout genre investit globalement et préférentiellement un des deux systèmes d'énonciation (discours ou récit) mis en évidence par E. Benveniste (1966). Il règle la place, le statut social des coénonciateurs. Il constitue un acte de parole global (informer/ expliquer/ convaincre/ polémiquer ...) qui vise à agir sur les représentations, les savoirs, les croyances du destinataire ;

- au niveau matériel : un genre sélectionne un médium spécifique (écrit/ oral/ visuel/ multimédia, hypertextuel...), ou une combinaison de ces médias, qui modèle l'énoncé (comme le montrent les travaux de médialogie de R. Debray);

- au niveau thématique: un genre impose des contraintes thématiques autorisant tel contenu thématique, en excluant d'autres ;

- au niveau formel : tout genre présente un plan de texte (conventionnel ou occasionnel (Adam, 1999: 69)) qui impose ou exclut des schémas séquentiels 
Margaret Bento

(narratifs/ descriptifs/ explicatifs/ argumentatifs/ dialogaux) articulés selon des enchaînements spécifiques à chaque genre ;

- au niveau stylistique : un genre opère, dans les sous-systèmes linguistiques composant une langue, des sélections lexicales, grammaticales. » (Richer, 2005, p. 69).

Cependant, pour cet auteur, cette notion des genres de discours doit être resituée dans un contexte plus large, pas uniquement textuel mais également actionnel où il y a primauté du sens sur la forme et où il y a un dépassement de la dimension langagière. Richer (2005) reprend à son compte le concept de "genres sociaux d'activités de Clot (1999, p. 174-175) qui ouvre la possibilité de penser conjointement le dire et le faire : "On ne constate pas seulement la présence d'énoncés déplacés ou, au contraire convenus dans un milieu social mais tout autant celle de gestes et d'actes matériels et corporels mal ou bien venus. De façon générale, on y trouve une gamme d'activités imposées, possibles ou prohibées. Les attendus sociaux d'un genre - souvent sousentendus - concernent autant les activités techniques et corporelles que les activités langagières. »

Pour Chini (2008, p. 10) il existe un flou dans le CECRL en ce qui concerne les choix à faire sur le plan des savoirs, savoir-faire et savoir-être à mettre en ouvre au niveau des contenus linguistiques. En effet, le Cadre indique bien que l'utilisateur est libre de choisir l'approche qu'il souhaite, même si «la typologie des actions langagières proposée par le cadre s'apparente, par certains côtés, au concept de « genre du discours» tel que Bronckart (1996) l'exploite dans la perspective d'interactionnisme socio-discursif qui est la sienne, ou encore à une classification des types de situations pragmatiques, dans la mesure où elle se fonde sur un ensemble riche de descripteurs étalonnés, organisés par activités langagières ou communicatives, qui détaillent les types de discours et de stratégies visés en tenant compte des aspects quantitatifs et qualitatifs des compétences linguistique, sociolinguistique et pragmatique attendues. » Pour l'auteure, cette démarche présentant « des listes détaillées de micro-fonctions, de formes grammaticales et de vocabulaire » conduit à faire des « listes fourre-tout » sans réflexion sur une progression possible et qui amènent les élèves à se centrer plus sur la tâche que sur les éléments linguistiques. Chini (2008) et Goutéraux (2008) proposent de mettre en place « des activités métalinguistiques et métacognitives » dans les classes de manière interactive dans une " logique de conceptualisation psycholinguistique » qu'elles définissent par ailleurs comme « un dépassement des spécificités de la tâche [...] de décontextualisation cognitive, de généralisation. » Pour Chini (2008, p. 16) la théorie de l'énonciation (d'inspiration culiolienne) répond aux exigences de la perspective actionnelle parce qu'elle " prend en compte la relation entre le langagier et le cognitif, dans la mesure où [elle] se donne comme objet non pas tant les systèmes linguistiques que l'activité de langage $[\ldots] »$ Pour l'auteure, c'est sur les procédures psycholinguistiques en jeu dans l'activité langagière qu'il faudrait cibler le travail réflexif en utilisant des concepts renvoyant à des opérations psycholinguistiques transversales (concepts de sujet, de relation, de repérage, de détermination...). Puisque la logique constructiviste impose que l'on parte des références de l'élève et donc de sa langue maternelle, l'élève peut ainsi transférer des éléments déjà connus pour accéder au sens de la langue cible, en l'occurrence des concepts énonciatifs communs aux deux langues.

Les quelques auteurs qui font référence aux sciences du langage dans le corpus convoquent soit la linguistique énonciative, soit les genres de discours en tentant de montrer en quoi ces phénomènes sont en adéquation avec l'approche actionnelle et certains éléments des théories d'apprentissage associées à cette perspective. Ainsi, les genres de discours dépassent la dimension uniquement langagière et on retrouve ici l'idée qu'une tâche se doit de prendre en compte à la fois les actions verbales et non verbales (§ 3.1.1.). La linguistique énonciative telle qu'elle est présentée par les auteures suggère la prise en compte de la métacognition et de la conceptualisation (§ 3.1.3. et 3.1.1.).

\section{Les théories sociologiques}

Bien que rarement convoquée en didactique des langues, la sociologie est pourtant présente dans le corpus à travers deux philosophes de la sociologie: Schütz (1998) avec le constructivisme phénoménologique et Habermas (1981) avec l'agir communicationnel. Leurs théories sont mises en résonance avec l'activité sociale et l'approche actionnelle.

Pour Schütz, l'action humaine est soumise au phénomène de socialisation. On retrouve ainsi les notions d'intersubjectivité et d'interactivité dans l'ap- 
propriation des connaissances mais aussi l'idée que les connaissances sont issues de la société et transmise par l'entourage (Perrichon 2009, p. 57 et ss.).

Pour Habermas, l'activité sociale est affaire de consensus entre les individus. L'agir communicationnel constitue la stratégie par laquelle on cherche à s'entendre avec l'autre, de façon à interpréter ensemble la situation et à s'accorder mutuellement sur la conduite à tenir (Springer, 2009, p. 27).

Les auteurs, en citant ces sociologues fondateurs de différents courants d'étude portant sur l'agir humain, montrent de leur point de vue l'influence et l'utilité de la communauté dans les apprentissages. Ces théories permettent d'étayer les descriptions des théories d'apprentissage retenues plus haut.

4. Synthèse

Ce travail de métasynthèse a tout d'abord permis de mettre en évidence la dominance des concepts liés aux théories de l'apprentissage dans la perspective actionnelle telle qu'elle est vue par les auteurs du corpus. Lorsque les théories linguistiques et sociologiques sont citées, elles s'articulent avec les théories de l'apprentissage. Ainsi l'agrégation des différentes notions évoquées par les chercheurs permet de constituer au final un puzzle cohérent autour de concepts constructivistes (Piaget), mais aussi et surtout socioconstructivistes (Vygotski, Bruner, Perret-Clermont, Doise et Mugny) où linguistiques énonciative et textuelle mais aussi théories de l'agir communicationnel (Habermas) et constructivisme phénoménologique (Schütz) se rencontrent.

Ainsi, on peut lister les caractéristiques de la perspective actionnelle en lien avec les théories d'apprentissage dominantes selon la communauté scientifique :

- à travers des projets/scénarios, les apprenants travaillent sur des tâches verbales et non-verbales, authentiques, qui doivent favoriser la réflexion sur la langue en présentant un problème à résoudre ;

- l'enseignant guide l'apprenant dans la résolution de problème ;

- les apprenants interagissent autour du problème afin de le résoudre ensemble ;

- les apprenants ont un retour réflexif sur leurs propres processus d'apprentissage.

En ce qui concerne les théories des sciences du langage, la faible quantité d'éléments relevés dans le corpus rend toute interprétation hasardeuse. Cependant deux phénomènes semblent se dégager : d'un côté les genres de discours au sens large (genres sociaux d'activités) et de l'autre la linguistique énonciative. Les auteurs justifient ces choix en les mettant en résonance avec certains principes liés aux théories de l'apprentissage de l'approche actionnelle : prise en compte de la dimension non-verbale dans les genres de discours, intégration de la conceptualisation et de la métacognition dans les analyses énonciatives.

Enfin pour la sociologie, l'évocation du constructivisme phénoménologique et de l'agir communicationnel sont mis en lien avec les concepts d'interaction et d'activité sociale.

5. Deux manières de comprendre la perspective actionnelle : le CECRL et les didacticiens

Afin de vérifier les liens qui peuvent être établis entre les résultats de cette recherche et les propositions du CECRL, les données de la méta-synthèse ont été croisées avec le CECRL.

Tout d'abord la notion de tâche est omniprésente à la fois dans le CECRL et dans le corpus (Conseil de l'Europe, 2001, p. 16). La tâche peut être authentique/réelle ou non et doit être prise dans ses dimensions langagières et extra-langagières. En revanche, la pédagogie du projet qui est vue dans le corpus comme le moyen à privilégier pour mettre en œuvre les tâches n'est considérée que comme un des moyens de cette mise en œuvre dans le CECRL, au même titre que les simulations globales, les jeux de rôle ou encore les exposés oraux (Conseil de l'Europe, 2001, p. 107, 119 ; Puren, 2009b). Il s'agit sans doute là d'un problème de définition car pour les auteurs du corpus la notion de projet recouvre une acception plus large que celle du Cadre.

L'idée de tâche associée à " un problème à résoudre » apparaît également dans la définition du CECRL mais elle se trouve accompagnée « d'une obligation à remplir et d'un but qu'on s'est fixé », ce qui élargit le champ des possibilités.

Il en est de même pour la réflexion sur la langue qui est présentée comme une des possibilités offertes aux apprenants parmi d'autres. Ainsi d'après le CECRL (Conseil de l'Europe, 2001, p. 107-108), certains théoriciens considèrent qu'il suffit d'être exposé à la langue pour être capable de l'utiliser et qu'il n'est pas nécessaire de l'enseigner ou de l'étudier explicitement ; d'autres postulent que les activités cognitives suffisent ; certains enfin, prétendent qu'on peut se contenter de la répétition d'exercices systématiques pour atteindre ce but. Le Cadre précise que « les utilisateurs du Cadre de référence envisageront et expliciteront selon le cas sur quelles hypothèses le 
Margaret Bento

travail relatif à l'apprentissage de la langue se fonde, et leurs conséquences méthodologiques. » (Conseil de l'Europe, 2001, p. 108).

En ce qui concerne le rôle de l'enseignant, encore une fois le CECRL laisse le champ libre : « Les utilisateurs du Cadre de référence envisageront et expliciteront selon le cas les responsabilités et rôles relatifs des enseignants et des apprenants dans l'organisation, la gestion, la conduite et l'évaluation du processus d'enseignement/apprentissage $[\ldots] »$ (Conseil de l'Europe, 2001, p. 110).

Quant au travail de groupe et à l'interaction, le CECRL y voit de nombreux avantages et l'encourage (Conseil de l'Europe, 2001, p. 99).

Le Cadre, tout comme les auteurs du corpus, incite au développement des stratégies métacognitives afin que les apprenants aient une meilleure connaissance de leur propre processus d'apprentissage en vue d'une plus grande autonomie (Conseil de l'Europe, 2001, p. 109 et 119).
Quant aux théories linguistiques et sociologiques, elles sont absentes du CECRL.

Le tableau ci-dessous récapitule les différentes caractéristiques liées aux théories tant au niveau du CECRL que du corpus.

Ce récapitulatif montre que les chercheurs et les didacticiens reprennent les idées d'approche actionnelle et de tâche telles qu'elles sont données dans le CECRL mais qu'ils les interprètent différemment. Ils infléchissent la perspective actionnelle et l'orientent vers les approches socioconstructivistes.

La liberté de choix qu'offre le CECRL dans les théories et méthodologies à mettre en ouvre dans l'enseignement/apprentissage des langues étrangères s'en trouve réduite. On peut aussi s'interroger sur ce « champ libre » laissé par le Cadre au regard du travail à accomplir par les enseignants et les auteurs des manuels. Quelle part d'influence ont le CECRL d'une part et les chercheurs et didacticiens d'autre part sur les pratiques enseignantes mais aussi sur les contenus des manuels?

Tableau 1 : Comparaison de la perspective actionnelle selon le CECRL et les auteurs du corpus

\begin{tabular}{|c|c|}
\hline CECRL & Auteurs du corpus \\
\hline $\begin{array}{l}\text { Tâche = " toute visée actionnelle que l'acteur se représente } \\
\text { comme devant parvenir à un résultat donné " }\end{array}$ & $\begin{array}{l}\text { Tâche = " toute visée actionnelle que l'acteur se représente } \\
\text { comme devant parvenir à un résultat donné » }\end{array}$ \\
\hline Si possible tâches authentiques, réelles & Si possible tâches authentiques, réelles \\
\hline Tâches langagières et extra-langagières & Tâches langagières et extra-langagières \\
\hline $\begin{array}{l}\text { Mise en œuvre des tâches dans la pédagogie du projet, les simu- } \\
\text { lations globales, les jeux de rôle... }\end{array}$ & Mise en œuvre des tâches dans la pédagogie du projet \\
\hline $\begin{array}{l}\text { L'exécution de la tâche conduit à une résolution de problème, une } \\
\text { obligation à remplir ou un but à atteindre }\end{array}$ & Exécution de la tâche conduit à une résolution de problème \\
\hline $\begin{array}{l}\text { Travail relatif à l'apprentissage de la langue ouvert (explicite ou } \\
\text { implicite, déductif ou inductif). }\end{array}$ & $\begin{array}{l}\text { Travail relatif à l'apprentissage de la langue inscrit dans une } \\
\text { logique de réflexion sur la langue }\end{array}$ \\
\hline Rien de préconisé au niveau du rôle de l'enseignant & Enseignant vu comme un guide pour l'apprenant \\
\hline $\begin{array}{l}\text { Interaction privilégiée parce que cela permet d'utiliser les } \\
\text { ressources langagières à différents niveaux }\end{array}$ & Interaction privilégiée pour résoudre un problème \\
\hline Incitation à la métacognition & Incitation à la métacognition \\
\hline Aucune théorie linguistique privilégiée & $\begin{array}{l}\text { "Théories des genres de discours » et linguistique énonciative } \\
\text { semblent privilégiés }\end{array}$ \\
\hline Aucune théorie sociologique privilégiée & $\begin{array}{l}\text { Les théories du constructivisme phénoménologique et de l'agir } \\
\text { communicationnel privilégiées }\end{array}$ \\
\hline
\end{tabular}




\section{CONCLUSION}

Cette métasynthèse a montré que les chercheurs en didactique des langues attribuent plusieurs concepts à la perspective actionnelle et ce malgré la volonté du Cadre de ne promouvoir aucune théorie en particulier. Ces concepts mis ensemble forment un tout cohérent et convergent vers le constructivisme et le socio-constructivisme donnant ainsi un cadre théorique à cette perspective tout en restreignant les possibilités offertes par le CECRL.

Reste à savoir si les enseignants et les auteurs de manuels prennent en compte ces concepts et s'ils le font, comment ils sont mis en œuvre dans les classes de langue et dans les manuels.

Cette recherche permet également d'interroger l'évolution de la didactique des langues étrangères en France. En effet, elle met en évidence le détachement que la didactique des langues étrangères a aujourd'hui avec la linguistique. Cela confirme le constat de Cuq et Gruca (2005, p. 139-140) : « [...] parmi les disciplines contributoires, la linguistique est incontestablement en perte de vitesse, et surtout ce que d'aucuns appellent son noyau dur (lexicologie, morphologie, syntaxe et même sémantique), et les nouvelles branches de cette discipline (acquisition, analyse de discours, etc.) ne compensent pas, au moins en quantité, ce recul. » Fortement marquée par les sciences du langage tout au long du vingtième siècle (méthodologies audio-orale, SGAV, communicative), l'analyse de notre corpus indique une didactique des langues dominée par la psychologie de l'éducation et les manières d'apprendre. Les didacticiens s'attachent plus particulièrement aux théories constructivistes et socioconstructivistes : attitude réflexive, métacognition, zone proximale de développement, étayage.

Par ailleurs, on a pu remarquer l'entrée discrète de la sociologie dans le panorama de l'approche actionnelle et voir comment elle s'articulait aux concepts des théories de l'apprentissage. La didactique des langues se définit notamment par les interactions et les intrications qu'elle entretient avec d'autres sciences et que «toute science peut contribuer à la didactique des langues, mais non s'y substituer. » (Defays, 2005, p. 167). 
Margaret Bento

\section{RÉFÉRENCES}

Altet M., (1997), Les pédagogies de l'apprentissage, Paris : PUF.

Bange P., Carol R. \& Griggs P., (2005), Lapprentissage d'une langue étrangère. Cognition et interaction, $\mathrm{Pa}$ ris : LHarmattan.

Bakhtine M., (1979), Les genres du discours, Esthétique de la création verbale, Paris: Gallimard.

Beaucher V. \& Jutras F., (2007), Étude comparative de la métasynthèse et de la méta-analyse qualitative. Recherches qualitatives, vol. 27 (2), p. 58-77.

Bronckart J.-P., (1996), Activité langagière, textes et discours : pour un interactionnisme socio-discursif. Lausanne : Delachaux \& Niestlé.

Bruner J., (1983), Le Développement de l'enfant : Savoir faire, savoir dire, Paris : Presses Universitaires de France.

Clot Y., (1999), La fonction psychologique, Paris : PUF.

Conseil de l'Europe, (2001), Cadre commun Européen de référence pour les langues, Paris : Didier.

Cuq J.-P. \& Gruca I., (2005), Cognitivisme et didactique des langues étrangères : de la référence à l'action pédagogique. Revue Parole, n 34/35/36, 137-163.

Defays J.-M., (2005), De l'enseignement à l'apprentissage : le paradigme cognitif en didactique des langues. Revue Parole, $n^{\circ}$ 34/35/36, 165-202.

Doise W. \& Mugny G., (1981), Le développement social de l'intelligence, Paris : InterEditions

Ellis R., (2003), Task-based language learning and teaching, Oxford : Oxford University Press.

Filliettaz L., (2002), La parole en action, Québec : Nota Bene.

Finfgeld D.L. (2003), Metasynthesis : the state of art - so far. Qualitative health research, (13)7, 893-904.

Gilly M., (2001), Interaction entre pairs et constructions cognitives : modèles explicatifs, in Gombert J.-E., (1990), Le développement métalinguistique, Paris: PUF.

Habermas J., (1981), Théorie de l'agir communicationnel, Paris : Fayard.
Jessen L., (2004), Extending meta-analysis. in S. Thorne (dir). Qualitative metasynthesis : reflections on methodological orientation and ideological agenda, Qualitative health research, (14)10, 1346-1347.

Maingueneau D., (1998), Analyser les textes de communication, Paris: Dunod.

Moscovici S., (1984), Le domaine de la psychologie sociale. Psychologie sociale, Paris : PUF.

Narcy-Combes J.-P. \& Walski J., (2004), Le concept de tâches soumis au crible de nouvelles questions. Les Cahiers de l'APLIUT, vol. XXIII (1), 27-44.

Noblit G. W. \& Hare D. R., (1988), Metaethnography : synthesinzing qualitative studies. London : Sage University Paper.

Nunan D., (2004), Task-based language teaching, Cambridge : Cambridge University Press.

Perret-Clermont A.-N., (1996), La construction de l'intelligence dans l'interaction sociale, Bern : Peter Lang,

Perret-Clermont A.-N. \& Nicolet M. (dir.) (2003), Interagir et connaître. Enjeux et régulations sociales dans le développement cognitif, Paris : L'Harmattan, 20-32, collection Figures de l'Interaction.

Puren C., (2006), Le Cadre Européen Commun de Référence et la réflexion méthodologique en didactique des langues-cultures : un chantier à reprendre. Synergies Europe, $\mathrm{n}^{\circ}$ 1, http://www.aplv-languesmodernes.org/ article.php3?id_article=35, (consulté le 21/01/2011).

Roulet E., (1999), La description de l'organisation du discours, Paris : Didier.

Roulet E., (2005), Un tournant actionnel en analyse et didactique des discours. Plurilinguisme et apprentissages, Mélanges Daniel Coste. Lyon : ENS.

Sandelowski M. \& Barroso J., (2006), Handbook for synthesizing qualitative research, New York: Springer $\mathrm{Pu}-$ blishing.

Schütz A., (1998), Éléments de sociologie phénoménologique, Paris : L'Harmattan.

Vygotski L. S. (1985 - 1re éd. en russe : 1934), Pensée et langage, Paris : Éditions Sociales. 


\section{Annexe : Littérature retenue et caractéristiques}

\begin{tabular}{|c|c|c|c|}
\hline Titres et sources & Auteurs & $\begin{array}{l}\text { Années de } \\
\text { publi. }\end{array}$ & Disciplines \\
\hline $\begin{array}{l}\text { CECR et dimension interculturelle de l'enseignement/apprentissage du FLE. } \\
\text { Quelles tâches possibles? Le français dans le monde, Recherches et applications, } \\
\mathrm{n}^{\circ} 45,102-110 \text {. }\end{array}$ & $\begin{array}{l}\text { Auger Nathalie et } \\
\text { Vincent Louis }\end{array}$ & 2009 & $\begin{array}{l}\text { Français langue } \\
\text { étrangère }\end{array}$ \\
\hline $\begin{array}{l}\text { Les tâches dans l'enseignement du FLE : rapport à la réalité et dimension didac- } \\
\text { tique, Le français dans le monde - Recherches et applications, } n^{\circ} 45,36-44 \text {. }\end{array}$ & Bérard Évelyne & 2009 & $\begin{array}{l}\text { Français langue } \\
\text { étrangère }\end{array}$ \\
\hline La démarche didactique en anglais - Du concours à la pratique, Paris, PUF & $\begin{array}{l}\text { Bourguignon } \\
\text { Claire }\end{array}$ & 2005 & $\begin{array}{l}\text { Anglais langue } \\
\text { étrangère }\end{array}$ \\
\hline $\begin{array}{l}\text { De l'approche communicative à l'« approche communic'actionnelle »: une } \\
\text { rupture épistémologique en didactique des langues-cultures, Synergie Europe, } \\
\mathrm{n}^{\circ} 1, \text { La richesse de la diversité : recherche et réflexions dans l'Europe des langues } \\
\text { et des cultures, 58-73 }\end{array}$ & $\begin{array}{l}\text { Bourguignon } \\
\text { Claire }\end{array}$ & 2006 & $\begin{array}{l}\text { Anglais langue } \\
\text { étrangère }\end{array}$ \\
\hline $\begin{array}{l}\text { Apprendre et enseigner les langues dans la perspective actionnelle : le scénario } \\
\text { d'apprentissage-action' Conférence donnée le } 7 \text { mars } 2007 \text { à l’Assemblée Générale de } \\
\text { la Régionale de l'APLV de Grenoble, http://www.aplv-languesmodernes.org/spip. } \\
\text { php?article } 865 \text { consulté le 21/01/2011 }\end{array}$ & $\begin{array}{l}\text { Bourguignon } \\
\text { Claire }\end{array}$ & 2007 & $\begin{array}{l}\text { Anglais langue } \\
\text { étrangère }\end{array}$ \\
\hline Enseigner l'anglais, Paris : Hachette Éducation & $\begin{array}{l}\text { Bourguignon } \\
\text { Claire }\end{array}$ & 2008 & $\begin{array}{l}\text { Anglais langue } \\
\text { étrangère }\end{array}$ \\
\hline $\begin{array}{l}\text { L'enseignement des langues à l'heure de la mondialisation : pour une pédago- } \\
\text { gie de l'action, Les cahiers du GERES, }{ }^{\circ} 1 \text {, http://geres.jimdo.com/les-cahiers- } \\
\text { du-geres/num\%C3\%A9ro-1/bourguignon-claire-l-enseignement-des-langues- } \\
\% \text { C3\%A0-l-heure-de-la-mondialisation-pour-une-p\%C3\%A9dagogie-de-l-action/ } \\
\text { consulté le 21/01/2011 }\end{array}$ & $\begin{array}{l}\text { Bourguignon } \\
\text { Claire }\end{array}$ & 2008 & $\begin{array}{l}\text { Anglais langue } \\
\text { étrangère }\end{array}$ \\
\hline $\begin{array}{l}\text { L'apprentissage des langues par l'action, L'approche actionnelle dans l'enseignement } \\
\text { des langues, Barcelone: Maison des langues, 49-78 }\end{array}$ & $\begin{array}{l}\text { Bourguignon } \\
\text { Claire }\end{array}$ & 2009 & $\begin{array}{l}\text { Anglais langue } \\
\text { étrangère }\end{array}$ \\
\hline Pour enseigner les langues avec le CECRL, Paris : Delagrave. & $\begin{array}{l}\text { Bourguignon } \\
\text { Claire }\end{array}$ & 2010 & $\begin{array}{l}\text { Anglais langue } \\
\text { étrangère }\end{array}$ \\
\hline $\begin{array}{l}\text { Perspective co-actionnelle et tice : quelles convergences pour l'enseignement de } \\
\text { la langue de spécialité ? Communication donnée dans le cadre des Journées d'Étude } \\
\text { de l'EA 2025-2-3 février } 2006 \text { http://www.langues-vivantes.u-bordeaux2.fr/frsa/ } \\
\text { pdf/CATROUX.pdf consulté le 21/01/201l. }\end{array}$ & Catroux Michèle & 2006 & $\begin{array}{l}\text { Anglais langue } \\
\text { étrangère }\end{array}$ \\
\hline $\begin{array}{l}\text { Portrait de l'enseignant en jardinier ou : Quelles perspectives de formation et de } \\
\text { recherche pour les migrants? Le français dans le monde, Recherches et applica- } \\
\text { tions, } \mathrm{n}^{\circ} 45,144-153 \text {. }\end{array}$ & $\begin{array}{l}\text { Champion } \\
\text { François }\end{array}$ & 2009 & $\begin{array}{l}\text { Français pour } \\
\text { les migrants }\end{array}$ \\
\hline $\begin{array}{l}\text { Approche actionnelle, plurilinguisme et conceptualisation linguistique, in } \\
\text { Danielle Chini et Pascale Goutéraux (éds), Psycholinguistique et didactique des } \\
\text { langues étrangères, Paris, Ophrys, 5-18. }\end{array}$ & Chini Danielle & 2008 & $\begin{array}{l}\text { Anglais langue } \\
\text { étrangère }\end{array}$ \\
\hline $\begin{array}{l}\text { Tâche, progression, curriculum, Le français dans le monde - Recherches et appli- } \\
\text { cations, } n^{\circ} 45,15-24 \text {. }\end{array}$ & Coste Daniel & 2009 & $\begin{array}{l}\text { Français langue } \\
\text { étrangère }\end{array}$ \\
\hline $\begin{array}{l}\text { Élaboration de tâches cognitives et énonciatives en langue étrangère, in Danielle } \\
\text { Chini et Pascale Goutéraux (éds), Psycholinguistique et didactique des langues } \\
\text { étrangères, Paris, Ophrys, 19-32. }\end{array}$ & $\begin{array}{l}\text { Goutéraux } \\
\text { Pascale }\end{array}$ & 2008 & $\begin{array}{l}\text { Anglais langue } \\
\text { étrangère }\end{array}$ \\
\hline $\begin{array}{l}\text { À propos de l'articulation entre l'agir de l'usage et l'agir de l'apprentissage dans } \\
\text { une approche actionnelle: une perspective sociocognitive, L'approche actionnelle } \\
\text { dans l'enseignement des langues, Barcelone: Maison des langues, 79-100. }\end{array}$ & Griggs Peter & 2009 & $\begin{array}{l}\text { Anglais LE }+ \\
\text { didactique des } \\
\text { langues }\end{array}$ \\
\hline $\begin{array}{l}\text { Travailler en projet dans la bande dessinée dans une approche actionnelle, Le } \\
\text { français dans le monde, Recherches et applications, } \mathrm{n}^{\circ} 45,54-61 .\end{array}$ & $\begin{array}{l}\text { Hamez Marie- } \\
\text { Pascale et } \\
\text { Brigitte Lepez }\end{array}$ & 2009 & $\begin{array}{l}\text { Français langue } \\
\text { étrangère }\end{array}$ \\
\hline $\begin{array}{l}\text { Tâches et coopération dans deux dispositifs universitaires de formation } \\
\text { à distance, Alsic, vol. } 6, \mathrm{n}^{\circ} 1,109-125 \text {. http://edutice.archives-ouvertes.fr/ } \\
\text { docs/00/00/18/36/HTML/ (consulté le 3/02/2011) }\end{array}$ & $\begin{array}{l}\text { Mangenot } \\
\text { François }\end{array}$ & 2003 & $\begin{array}{l}\text { Français langue } \\
\text { étrangère }\end{array}$ \\
\hline
\end{tabular}




\begin{tabular}{|c|c|c|c|}
\hline $\begin{array}{l}\text { Internet, tâches et vie réelle, Le français dans le monde, Recherches et applica- } \\
\text { tions, } n^{\circ} 45,82-90\end{array}$ & $\begin{array}{l}\text { Mangenot } \\
\text { François et } \\
\text { Frédérique } \\
\text { Penilla }\end{array}$ & 2009 & $\begin{array}{l}\text { Français langue } \\
\text { étrangère }\end{array}$ \\
\hline $\begin{array}{l}\text { L'enseignement-apprentissage des langues : un agir ensemble qui s'affirme, pour } \\
\text { le site APVL-LanguesModernes. org http://www.aplv-languesmodernes.org/spip. } \\
\text { php?article2168 consulté le 30/01/2011 }\end{array}$ & $\begin{array}{l}\text { Médioni } \\
\text { Marie-Alicia }\end{array}$ & 2008 & $\begin{array}{l}\text { Didactique des } \\
\text { langues }\end{array}$ \\
\hline $\begin{array}{l}\text { Situations d'apprentissage et activité des élèves en langues vivantes étrangères. } \\
\text { Analyse d'une mise en situation en espagnol, Thèse de doctorat sous la direction } \\
\text { de Jean-Yves Rochex, Paris, Université de Paris } 8 .\end{array}$ & $\begin{array}{l}\text { Médioni } \\
\text { Marie-Alicia }\end{array}$ & 2009 & $\begin{array}{l}\text { Didactique de } \\
\text { l'espagnol }\end{array}$ \\
\hline 25 pratiques pour enseigner les langues, Lyon : Chroniques sociales & $\begin{array}{l}\text { GFEN : Marie- } \\
\text { Alicia Médioni }\end{array}$ & 2010 & $\begin{array}{l}\text { Didactique des } \\
\text { langues }\end{array}$ \\
\hline $\begin{array}{l}\text { Apprendre une langue en ligne dans une perspective actionnelle. Effets de l'inte- } \\
\text { raction sociale, Thèse de doctorat sous la direction de Michèle Kirch, Strasbourg, } \\
\text { Université de Strasbourg l }\end{array}$ & Nissen Elke & 2003 & $\begin{array}{l}\text { Science de } \\
\text { l'éducation }\end{array}$ \\
\hline $\begin{array}{l}\text { Modalité d'interaction humaine dans la formation en ligne : son influence sur } \\
\text { l'apprentissage, Savoirs, } \mathrm{n}^{\circ} \text { 8, p. 87-106. }\end{array}$ & Nissen Elke & 2005 & $\begin{array}{l}\text { Science de } \\
\text { l'éducation }\end{array}$ \\
\hline $\begin{array}{l}\text { Mettre en œuvre une approche interactionnelle sur le Web 2.0, L'approche action- } \\
\text { nelle dans l'enseignement des langues, Barcelone : Maison des langues, 263- } 285\end{array}$ & $\begin{array}{l}\text { Ollivier } \\
\text { Christian }\end{array}$ & 2009 & $\begin{array}{l}\text { Didactique du } \\
\text { FLE }\end{array}$ \\
\hline $\begin{array}{l}\text { Agir d'usage et agir d'apprentissage en didactique des langues-cultures étran- } \\
\text { gères : enjeux conceptuels, évolution historique et construction d'une nouvelle } \\
\text { perspective actionnelle, Thèse de doctorat sous la direction de Christian Puren, } \\
\text { Saint-Etienne : Université Jean Monnet. }\end{array}$ & Perrichon Émilie & 2008 & $\begin{array}{l}\text { Didactique des } \\
\text { langues }\end{array}$ \\
\hline $\begin{array}{l}\text { Perspective actionnelle et pédagogie du projet: De la culture individuelle à la } \\
\text { construction d'une culture d'action collective, Synergies des Pays Riverains de la } \\
\text { Baltique, } n^{\circ} 6,91-111\end{array}$ & Perrichon Émilie & 2009 & $\begin{array}{l}\text { Didactique des } \\
\text { langues }\end{array}$ \\
\hline $\begin{array}{l}\text { Perspectives actionnelles et perspectives culturelles en didactique des langues- } \\
\text { cultures : vers une perspective co-actionnelle co-culturelle, Les langues modernes, } \\
n^{\circ} 3 / 2002,55-71\end{array}$ & Puren Christian & 2002 & $\begin{array}{l}\text { Didactique des } \\
\text { langues }\end{array}$ \\
\hline $\begin{array}{l}\text { De l'approche par les tâches à la perspective co-actionnelle, } \\
\text { Les Cahiers de l'APLIUT (revue de l'Association des Professeurs de langues des } \\
\text { Instituts Universitaires de Technologie), vol. XXIII, } \mathrm{n}^{\circ} 1,10-26\end{array}$ & Puren Christian & 2004 & $\begin{array}{l}\text { Didactique des } \\
\text { langues }\end{array}$ \\
\hline $\begin{array}{l}\text { Explication de textes et perspective actionnelle : la littérature entre le dire } \\
\text { scolaire et le faire social, site APLV }\end{array}$ & Puren Christian & 2006 & $\begin{array}{l}\text { Didactique des } \\
\text { langues }\end{array}$ \\
\hline $\begin{array}{l}\text { Enseigner et apprendre les langues sur un mode qui change avec le monde : } \\
\text { l'exemple du passage de l'approche communicative à la perspective actionnelle, } \\
\text { Conférence au Seminario Nazionale LEND (Lengua e nuova didattica), Bologna } \\
\text { 18-19-20 ottobre 2007, Insegnare e apprendere le lingue in un mondo che cambia }\end{array}$ & Puren Christian & 2007 & $\begin{array}{l}\text { Didactique des } \\
\text { langues }\end{array}$ \\
\hline $\begin{array}{l}\text { Variations sur la perspective de l'agir social en didactique des langues-cultures } \\
\text { étrangères, Le français dans le monde, Recherches et applications, n } 45,154-168\end{array}$ & Puren Christian & $2009 a$ & $\begin{array}{l}\text { Didactique des } \\
\text { langues }\end{array}$ \\
\hline $\begin{array}{l}\text { Entre l'approche communicative et la perspective actionnelle, quoi de neuf ?, } \\
\text { Abdelgaber Sylvie \& Médioni Maria-Alice (dir.), Les Cahiers Pédagogiques, } \\
\text { Collection des HNS hors-séries numériques, } n^{\circ} 18,87-91\end{array}$ & Puren Christian & $2009 b$ & $\begin{array}{l}\text { Didactique des } \\
\text { langues }\end{array}$ \\
\hline $\begin{array}{l}\text { La nouvelle perspective actionnelle et ses implications sur la conception des } \\
\text { manuels de langue, Lapproche actionnelle dans l'enseignement des langues, } \\
\text { Barcelone: Maison des langues, 119-137 }\end{array}$ & Puren Christian & $2009 c$ & $\begin{array}{l}\text { Didactique des } \\
\text { langues }\end{array}$ \\
\hline $\begin{array}{l}\text { Mises au point de/sur la perspective actionnelle, http://www.christianpuren. } \\
\text { com/mes-travaux-liste-et-liens/201le/ }\end{array}$ & Puren Christian & 2011 & $\begin{array}{l}\text { Didactique des } \\
\text { langues }\end{array}$ \\
\hline $\begin{array}{l}\text { De l'approche communicative à l'approche actionnelle : apports du Cadre euro- } \\
\text { péen commun de référence pour les langues, conférences au CIEP, http://sites. } \\
\text { google.com/site/seminairebogota2006/del\%E2\%80\%99approchecommunicative } \\
\% \text { C3\%A01\%E2\%80\%99approcheactionnelle : consulté le 31/01/2011 }\end{array}$ & Riba Patrick & 2006 & $\begin{array}{l}\text { Français langue } \\
\text { étrangère }\end{array}$ \\
\hline $\begin{array}{l}\text { Conception et validité de tâches évaluatives dans une perspective actionnelle, Le } \\
\text { français dans le monde, Recherches et applications, } \mathrm{n}^{\circ} 45,124-132\end{array}$ & Riba Patrick & 2009 & $\begin{array}{l}\text { Français langue } \\
\text { étrangère }\end{array}$ \\
\hline
\end{tabular}




\begin{tabular}{|c|c|c|c|}
\hline $\begin{array}{l}\text { Le Cadre européen commun de référence pour les langues: Des perspectives } \\
\text { d'évolution méthodologique pour l'enseignement/apprentissage des langues?, } \\
\text { Synergies Chine, } n^{\circ} 5.63-71\end{array}$ & $\begin{array}{l}\text { Richer } \\
\text { Jean-Jacques }\end{array}$ & 2005 & $\begin{array}{l}\text { Français langue } \\
\text { étrangère }\end{array}$ \\
\hline $\begin{array}{l}\text { Lectures du Cadre : continuité ou rupture ? L'approche actionnelle dans l'enseigne- } \\
\text { ment des langues, Barcelone, Maison des langues, 13-48. }\end{array}$ & $\begin{array}{l}\text { Richer } \\
\text { Jean-Jacques }\end{array}$ & 2009 & $\begin{array}{l}\text { Français langue } \\
\text { étrangère }\end{array}$ \\
\hline $\begin{array}{l}\text { La perspective actionnelle et l'approche par les tâches, Le français dans le monde } \\
\text { - Recherches et applications, } n^{\circ} 45,6-14\end{array}$ & Rosen Évelyne & 2009 & $\begin{array}{l}\text { Français langue } \\
\text { étrangère }\end{array}$ \\
\hline $\begin{array}{l}\text { Scénarios TICE et perspective actionnelle : la norme mise à mal ?, in Olivier } \\
\text { Bertrand et Isabelle Schaffner, Quel français enseigner ? La question de la norme } \\
\text { dans l'enseignement /apprentissage, Paris, Les éditions de l'école polytechnique, } \\
73-94\end{array}$ & Soubrié Thierry & 2009 & $\begin{array}{l}\text { Français langue } \\
\text { étrangère }\end{array}$ \\
\hline $\begin{array}{l}\text { La dimension sociale dans le CECR : pistes pour scénariser, évaluer et valoriser } \\
\text { l'apprentissage collaboratif, Le français dans le monde - Recherches et applications, } \\
n^{\circ} 45,25-34\end{array}$ & Springer Claude & 2009 & $\begin{array}{l}\text { Français langue } \\
\text { étrangère }\end{array}$ \\
\hline
\end{tabular}

